\title{
WARPED ACCRETION DISKS AND LONGTERM X-RAY PERIODICITIES
}

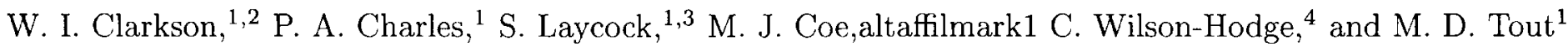

\section{RESUMEN}

En la actualidad se conoce un numero significativo de binarias de rayos $\mathrm{X}$ que presentan periodicidades de largo plazo en escalas de tiempo de $\sim 10$ - 100 días. Fueron propuestos varios mecanismos físicos que dan origen a tales periodicidades, uno de los cuales es alabeo empujado por la radiación y precesión del disco de acreción . Recientes trabajos teóricos predicen la estabilidad del alabeo del disco como una función del cociente de masa, radio binario, viscosidad y eficiencia de acreción. Investigamos la estabilidad de las periodicidades superorbitales en las binarias de rayos X de estrellas de neutrones Cyg X-2, LMC X-4, SMC X-1 y Her X-1, confrontando las predicciones de estabilidad con las observaciones. Encontramos que el periodo y la naturaleza de las variaciones superorbitales son consistentes en estas fuentes con las predicciones de la teoría de distorsión.

\section{ABSTRACT}

A significant number of X-ray binaries are now known to exhibit long-term periodicities on timescales of $\sim 10$ 100 days. Several physical mechanisms have been proposed that give rise to such periodicities, one of which is radiation-driven warping and precession of the accretion disk. Recent theoretical work predicts the stability to disk warping as a function of the mass ratio, binary radius, viscosity and accretion efficiency. We investigate the stability of the superorbital periodicities in the neutron star X-ray binaries Cyg X-2, LMC X-4, SMC X-1 and Her X-1, and thereby confront stability predictions with observation. We find that the period and nature of the superorbital variations in these sources is consistent with the predictions of warping theory.

Key Words: ACCRETION, ACCRETION DISKS - X-RAYS: BINARIES

\section{INTRODUCTION}

In $\sim 20$ bright X-ray binaries $(\mathrm{XRB})$ a third (or "superorbital") period is present in addition to the usual periodicities. Broadly speaking, superorbital variations divide into two observational classes, with clear, stable X-ray intensity variations in the $\sim 30$ day range forming one category (e.g. SMC X-1, Her $\mathrm{X}-1$, LMC X-4), and more quasi-periodic, longer periodicities (as with Cyg X-2) in the $\sim 50-200$ day range forming the other (see Clarkson et al 2003a, hereafter Paper I, and references therein). Most superorbital periodicities have been interpreted as a precessing, possibly warped accretion disk, motivating intense recent theoretical examination of the mechanisms for driving and maintenence of such a warp. The most popular model is warp driving through the interception and re-emission of radiation from the compact object (Ogilvie and Dubus 2001, hereafter OD01, and references therein). For reasonable values of the global disk viscosity and accretion efficiency, OD01 predict the stability of an accretion disk to warping from the binary separation $r_{b}$ and

\footnotetext{
${ }^{1}$ University of Southampton.

${ }^{2}$ Open University.

${ }^{3}$ HArvard Centre for Astrophysics.

${ }^{4}$ NASA/MSFC.
}

mass ratio $q=\frac{M_{2}}{M_{1}}$, giving a further observational constraint. Two viable alternatives to the radiationdriving scenario are (i) warp driving by an accretion disk wind (Schandl 1996) and (ii) driving through the interaction of sheet currents on the disk with the magnetic field of the donor or accretor (Murray $2000 \&$ references therein). We therefore examine the stability predictions of radiation-driven warping framework with observations of longterm periodicities in four persistently bright X-ray Binaries. We summarise the major results of the work here and refer the reader to Paper I and Clarkson et al 2003b (hereafter Paper II) for full details and references.

\subsection{Sources Selected}

Four bright XRB were chosen, where for each source (i) evidence already exists that a precessing, warped accretion disk is present and (ii) the binary parameters $q$ and $r_{b}$ are known. Her X-1 and LMC $\mathrm{X}-4$ are predicted by OD01 to exhibit single-mode periodicities, which should be stable over long time periods. Cyg X-2 is deep within the region of parameter space in which multimode variation is expected. SMC X-1 is on the border between the regions, suggesting a competition of modes may be possible (See OD01 for more on these predictions). 


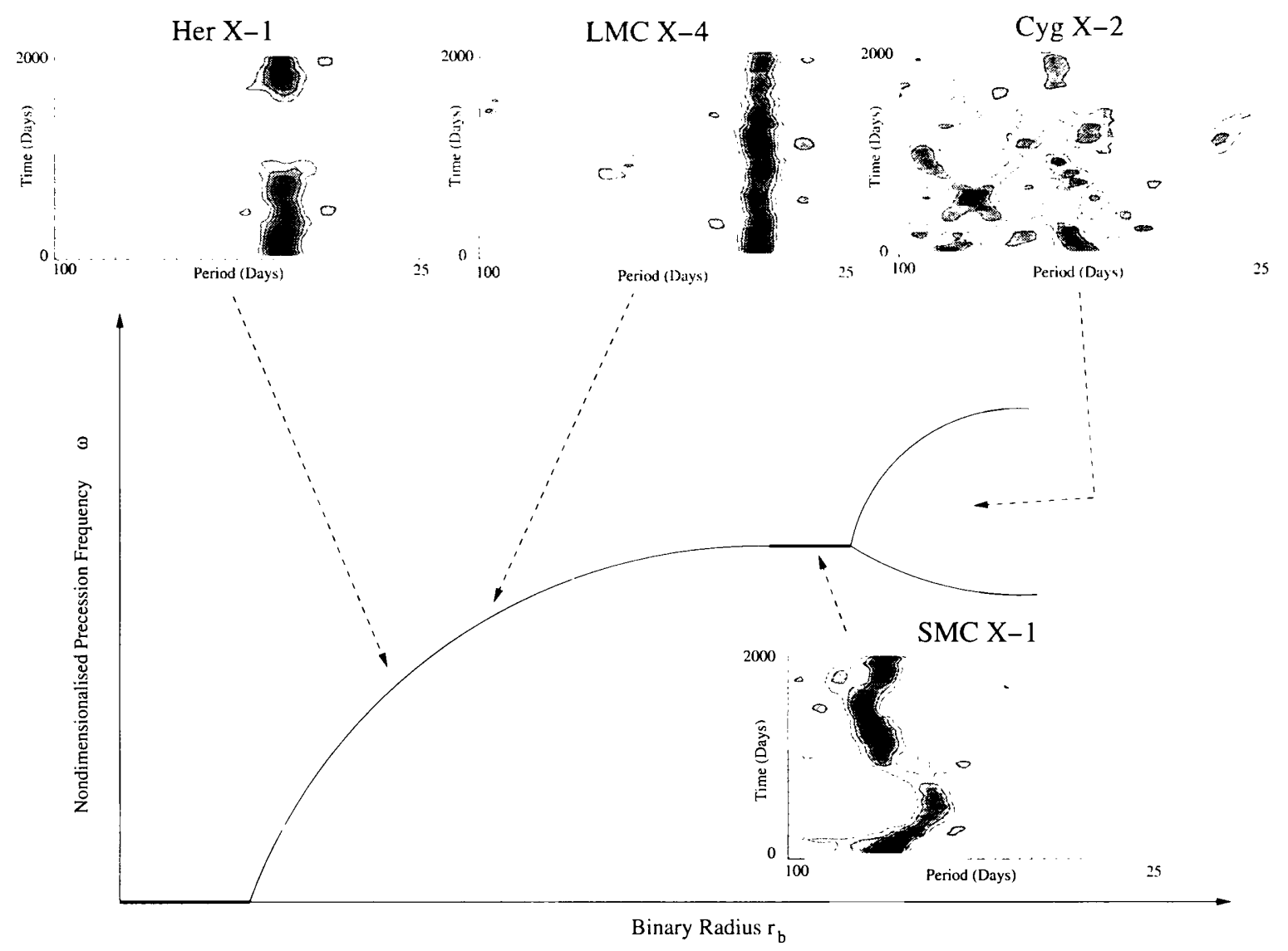

Fig. 1. Schematic bifurcation diagram. As the control parameter $r_{b}$ is increased, precession solutions evolve from stable mode 0 warping to a situation with two or more possible warping modes. Near the mode region border, the solution becomes marginally unstable as short-lived mode- 1 instabilities form

\section{STABILITY OF X-RAY SUPERORBITAL PERIODICITIES}

The time evolution of superorbital periodicities from the four sources were traced using dynamic power-spectral analysis of the superb longterm datasets of RXTE/ASM. With their stable behaviour in the DPS and simple superorbital lightcurves, Her X-1 and LMC X-4 show clear evidence for mode 0 warping. SMC X-1 shows a simple superorbital fold that varies over time, probably due to disk instabilities brought about as other modes begin to form (Paper I). Cyg X-2 shows at least two independent modes of warping, thought to represent mode 0,1 and possibly higher warp modes.

The sources thus behave in ways qualitatively predicted by the stability analysis of OD01. Her X1 and LMC X-4 are both predicted to show stable mode 0 warping, which is indeed observed. SMC X-1 is thought to show warping commensurate with its position near the mode 0 and mode 1 regions. Cyg $\mathrm{X}-2$ is deep within the region predicting mode 1 and higher modes, which is consistent with the appearance and interaction of at least two warping modes.
The commensurability of our results with the predictions of radiation-driven warping suggest this mechanism is at work in these high-luminosity systems. Magnetic warping and wind-driving do not predict such agreement: the magnetic field of the donor is largely independent of $r_{b}$ and $q$, whereas in the disk wind case the predictions are incomplete, as physically correct characterisation of the effects of a disk wind have yet to be established (OD01).

WIC and SL were in receipt of PPARC studentships. This work was only possible through the efforts of the RXTE/ASM teams at MIT and NASA/GSFC.

\section{REFERENCES}

Clarkson, W. I., Charles, P. A.. Coe, M. J., Laycock, S.. Tout, M. D.,Wilson, C. A. 2003a MNRAS 339, 44 T

Clarkson, W. I., Charles, P. A.. Coe. M. J.. Laycock. S. 2003b MNRAS 343, 1213

Murray, J.R., Chakrabarty, D.. Wynn. G.A.. Kramer. L. 2002 MNRAS 335, 2.17

Ogilvie, G.I., Dubus, G. 2000, MNRAS. 320, 45:

Schandl, S. 1996, Ac.A. 307. 95 